\title{
Pathologic and Treatment Outcomes Among a Geriatric Population of Endometrial Cancer Patients: An NRG Oncology/ Gynecologic Oncology Group Ancillary Data Analysis of LAP2
}

\author{
Erin A. Bishop, MD ${ }^{1}$, James J. Java, PhD $^{2}$, Kathleen N. Moore, MD ${ }^{3}$, and Joan L. Walker, \\ $M^{3}$ \\ ${ }^{1}$ University of Oklahoma, Department of Obstetrics and Gynecology, Section of Gynecologic \\ Oncology, Oklahoma City, OK \\ ${ }^{2}$ NRG Oncology Satistics and Data Center, Roswell Park Cancer Institute; Buffalo, NY \\ ${ }^{3}$ Stephenson Oklahoma Cancer Center at the University of Oklahoma, 800 NE $10^{\text {th }}$ Street, \\ Oklahoma City, OK 73121
}

\begin{abstract}
Objectives-Elderly endometrial cancer patients have worse disease specific survival than their younger counterparts, but the cause for this discrepancy is unknown. The goal of this analysis is to compare outcomes by age in a fully staged elderly endometrial cancer population.

Methods/Materials-This is an analysis of patients on GOG LAP2 which included clinically early stage endometrial cancer patients randomized to laparotomy vs. laparoscopy for surgical staging. Patients were divided into risk groups based on criteria defined by GOG-99. Differences in outcomes and adjuvant therapy were assessed within these risk groups.
\end{abstract}

Results-LAP2 included 715 patients $\geq 70$ years old (yo). With increasing age, worse tumor characteristics were seen. Older patients received similar rates of adjuvant therapy when stratified by stage. Patients $\geq 70$ yo had significantly worse progression-free survival (PFS) and overall survival (OS) and on multivariate analysis older age as well as high risk uterine factors were predictors of PFS and OS while stage and lymph node metastasis were not. When patients were divided into GOG-99 risk categories, the majority of those who met high-intermediate risk criteria did so based on age $>70$ yo and grade 2- 3 disease. These patients had low risk of recurrence (3.3\%) compared to those who met criteria by age $>70$ yo and all 3 uterine factors (20.9\%).

Conclusions-In early stage endometrial cancer, patients $\geq 70$ yo who undergo similar surgical management and adjuvant therapy, age as well as tumor characteristics independently predict recurrence. Most patients > 70yo meet high-intermediate risk criteria for recurrence based on age and one other uterine risk factor and our results suggest these patients are at low risk for recurrence.

Corresponding author: Erin A. Bishop, MD, Medical College of Wisconsin, Division of Gynecology Oncology, 9200 West Wisconsin Avenue, Milwaukee, Wisconsin 53226, erbishop@ mcw.edu, phone: 414-805-6623, Fax: 414-805-6622.

james.j.java@gmail.com, kathleen-moore@ouhsc.edu; joan-walker@ouhsc.edu

This was presented in part at the 44th Annual Meeting of the Society of Gynecologic Oncologists, March 9-12, 2013, Los Angeles, CA. 


\section{INTRODUCTION}

Endometrial cancer, a disease that generally occurs in post-menopausal women, is expected to increase in prevalence as the population ages. In 2015, there were over 52,000 new cases of endometrial cancer diagnosed in the United States with over 8,500 deaths. While only $25 \%$ of cases are diagnosed in women older than 75 years old (yo), $50 \%$ of deaths due to endometrial cancer occur in these patients[1],[2]. Potential factors that cause this difference in incidence and mortality include more aggressive tumors in elderly women, less aggressive therapy for elderly patients, and age is a poor prognostic marker for endometrial cancer.

Previous studies show that age plays a role in predicting recurrence in endometrial cancer. Gynecologic Oncology Group Study (GOG)-99 was designed to compare surgery alone (total abdominal hysterectomy, bilateral salpingo-oophorectomy including lymphadenectomy) versus surgery and adjuvant radiotherapy (RT) in patients with stages IB, IC, and occult stage II endometrioid endometrial cancer. There was a significant decrease in recurrence rates ( $12 \%$ vs $3 \%$ ) in patients treated with postoperative RT, but no difference in overall survival (OS). A subset analysis identified a group with a $25 \%$ risk of recurrence. This high intermediate risk (H-IR) group is identified by age plus uterine risk factors including grade 2 or 3 , presence of lymph vascular space invasion (LVSI), and depth of invasion to the outer $1 / 3$ of the myometrium. The GOG-99 algorithm identified H-IR patients as age $>70$ yo +1 uterine factor, age 50-70yo +2 factors, or any age +3 factors [3]. Other studies such as the first Post-Operative Radiotherapy in Endometrial Cancer (PORTEC-1) trial also found age was associated with increased disease recurrence [4]. In 1996 the GOG started a randomized prospective trial (GOG-2222 or LAP2) to compare comprehensive surgical staging by laparotomy versus laparoscopy for the treatment of clinical stage I to IIA uterine cancer $(\mathrm{n}=2616)$. The GOG has published results of LAP2 regarding surgical outcomes, completeness of surgical staging, recurrence free survival, and quality of life (QoL).[5],[6] LAP2 is one of the largest prospective trials looking at outcomes in clinically early stage endometrial cancer and has 715 patients $\geq 70$ yo. This allows for retrospective assessment of a large subset of elderly patients with clinically early stage endometrial cancer.

The goal of this ancillary review is to report the surgico-pathologic findings, recurrence, and survival outcomes in patients $\geq 70$ versus $<70$ yo who participated in GOG LAP2. The results of this study will further elucidate the etiologies of the discrepancies seen in incident cases of, and mortality due to, endometrial cancer in elderly patients. GOG LAP2 included patients with primarily early stage disease and good performance status and required complete surgical staging, so differences in outcomes should be attributable to disease and postoperative therapy.

\section{MATERIALS AND METHODS}

This is an analysis of patients enrolled on GOG LAP2. The details of inclusion and exclusion criteria can be seen in the original manuscript [5]. The study was designed to compare laparoscopy with laparotomy for the purpose of complete comprehensive surgical staging of uterine cancer. The primary outcome of the study was recurrence-free survival. 
Other end points included perioperative adverse events, quality of life, and survival. Eligibility requirements were clinical stage I to IIA uterine cancer, adequate bone marrow, renal, and hepatic function, and GOG performance status less than 4.

The initial analysis in this retrospective study involved comparing adjuvant therapy and outcomes by age in the entire LAP2 population. Subsequently, patients were divided into risk groups defined by GOG protocol 99 based on pathologic findings. The GOG 99 H-IR group included stage I and occult stage II patients with endometrioid histology who met the criteria listed in the introduction. In order to reflect updated staging from 2009, depth of invasion $>50 \%$ was used whereas in GOG protocol $99 \mathrm{H}-\mathrm{IR}$ was defined as outer third invasion. In patients $>70 \mathrm{yo}$, recurrence rates were compared between patients who met H-IR criteria by age and additional uterine risk factors. Differences in outcomes and adjuvant therapy were assessed within these risk groups. Categorical variables were compared between subgroups by the Pearson chi-square test, and continuous variables by the Wilcoxon-Mann-Whitney test or the Kruskal-Wallis test.[7-9] Survival was estimated using the Kaplan-Meier method [10].

The Cox proportional hazards model was used to evaluate independent prognostic factors (including age, race/ethnicity, BMI, FIGO stage, tumor grade, performance status, lymphovascular invasion, and nodal metastasis), without any variable selection, and to estimate their covariate-adjusted effects on survival [11]. The nonlinearity of the effect of continuous variables was assessed using restricted cubic splines [12] and multicollinearity was checked through the method of variance inflation factors (VIF) [13]. All statistical tests were two-tailed with the significance level set at $\alpha=0.05$. Statistical analyses were performed using $\mathrm{R}$ programming language and environment [14].

\section{RESULTS}

\section{Total LAP2 population uterine characteristics and adjuvant treatment}

A total of 2516 patients were included in this analysis based on LAP2 eligibility. When the total LAP2 population was divided into age by decade, 555 patients $(21.2 \%)$ were $70-79$ yo and 160 patients $(6.4 \%)$ were $\geq 80 \mathrm{yo}$. With increasing age there were increased rates of serous histology $(\mathrm{p}<0.001)$, stage 1B versus stage 1A (FIGO 2009 staging [15]) and overall higher stage disease $(p<0.001)$, grade 3 tumors $(p<0.001)$, and rates of positive LVSI $(\mathrm{p}<0.001)$ (Table 1). The majority of patients on LAP2 had endometrioid or serous histology, so only these histologies are shown but all histological subtypes were included in the analysis. When comparing postoperative therapy, rates of chemotherapy were similar across age groups ranging between 8.8 and $13.5 \%(\mathrm{p}=0.122)$, while rates of radiation increased with increasing age until age 79 ranging from $20 \%$ in patients $<50$ yo to $30.6 \%$ in patients $70-79$ yo at which point a drop in the percent of patients 280 yo treated with adjuvant radiation was seen $(23.8 \%$; $\mathrm{p}=0.001)$. Rates of receiving any adjuvant therapy increased with increasing age until age 79 ranging from $22.8 \%$ in patients $<50$ yo to $36.9 \%$ in patients $70-79 y o$ at which point a drop in receiving any adjuvant therapy was seen in patients 280 yo $(29.4 \%$; $p<0.001)$ (Table 1). However, when looking at rates of adjuvant therapy by age and stage there does not appear to be a clear tendency towards older patients receiving more or less adjuvant therapy (Table 1 and Table 2: supplemental Digital Data); this is confirmed by 
an application of the Woolf test for interaction to the age vs. therapy contingency tables stratified by stage ( $\mathrm{P}=0.800$, NS), followed by the Cochran-Mantel-Haenszel test for conditional independence $(\mathrm{P}=0.232$, NS).

\section{Recurrence and survival in total LAP2 population}

In univariate analysis, recurrence rates increased significantly with age with $4.9 \%$ of patients $<50$ yo recurring compared to $14.4 \%$ in patients $\$ 80$ yo ( $<<0.001$ ). When looking at cause of death, $1.5 \%$ of $<50 \mathrm{yo}$ died of disease compared to $16.5 \%$ in patients $\geq 80 \mathrm{yo}$. Patients $\geq 80 \mathrm{yo}$ also died more often of causes unrelated to disease than patients $<50$ yo $(23.4 \%$ vs. $1.1 \%$; $\mathrm{p}<0.001$ ) (Table 1). The 70 mo. progression-free survival (PFS) for $<70$ yo vs. $\geq 70$ is $85 \%$ vs. $65 \%$ (p<0.001). The 70 mo OS for patients $<70$ vs. $\geq 70$ is $89 \%$ vs. $69 \%(p<0.001)$ (Figure 1).

On multivariate analysis the factors significant for PFS were depth of invasion $250 \%$ (hazard ratio $[\mathrm{HR}]=2.90,95 \%$ CI: $1.46-5.74, \mathrm{p}=0.002$ ), grade 3 disease (HR 2.14, 95\% CI: $1.37-$ 3.34; $\mathrm{p}<0.001$ ), serous histology (HR 1.59, 95\% CI: 1.17-2.16; $\mathrm{p}=0.003$ ), presence of LVSI (HR 1.41, 95\% CI: 1.11-1.81; $\mathrm{p}=0.005$ ), and age (HR 1.04, 95\% CI: $1.03-1.05 ; \mathrm{p}<0.001$ ). For OS these same factors remained significant with the addition of non-white race (HR $1.55,95 \%$ CI: 1.12-2.14; $\mathrm{p}=0.008$ ) and body mass index (BMI) (HR 1.02, 95\% CI: 1.00 1.04; $\mathrm{p}=0.025)$. Stage, lymph node metastasis, performance status, and receiving postoperative therapy were not independent predictors for PFS or OS.

\section{GOG99 H-IR population adjuvant treatment}

When patients were divided into risk groups based on GOG-99 H-IR criteria, 37\% (926) of the total LAP2 population met GOG-99 H-IR criteria, $43 \%$ of these patients were $\geq 70$ yo. While only $28 \%$ of the LAP2 population was $\geq 70$ yo, $55 \%$ met GOG- 99 H-IR criteria. Of the $926 \mathrm{H}-$-IR patients, data about adjuvant therapy existed for $96 \%$ (892). A total of $28 \%$ received adjuvant treatment: $96 \%$ radiation, $5.2 \%$ chemotherapy and radiation, and $4 \%$ chemotherapy alone. When looking at rates of adjuvant treatment across age groups in this H-IR subset, as age increased, significantly less radiation was received with $60 \%$ of patients $<50$ yo receiving radiation vs. $24-27 \%$ in patients $50-79$ yo and only $17.3 \%$ in patients 280 yo $(\mathrm{p}=0.011)$ (Table 3). There is also a trend towards less treatment in older patients, but when the 15 patients <50yo are excluded; similar rates of no adjuvant therapy are seen across remaining age groups with a range of $68.5 \%$ to $71.6 \%$.

\section{Recurrence and survival in GOG99 H-IR population}

There are similar rates of disease recurrence across the age groups ranging from 4.3-20\% $(\mathrm{p}<0.128)$, but older patients have higher death rates $(\mathrm{p}<0.001)$. The main discrepancy in cause of death between age groups is causes unrelated to disease or treatment with $3.4 \%$ of patients 60-69yo dying of causes unrelated to disease vs. $13.9 \%$ in patients 280 yo. Death from disease is generally low, ranging from $3.1 \%$ to $7.6 \%$ (Table 3 ).

When looking at recurrence rates in the entire GOG-99 H-IR group, recurrence rates were significantly higher in the H-IR patients who received adjuvant therapy compared to the $\mathrm{H}$ IR patients who received no adjuvant therapy $(9.6 \%$ versus $4.4 \%$; $\mathrm{p}=0.003)$ with no 
significant difference in death rates between these groups $(\mathrm{p}=0.20)$ (Table 4). Multivariate analysis of GOG-99 H-IR patients in LAP2 shows no difference in PFS and OS between treated vs. untreated patients (p-values 0.83 and 0.80 respectively).

On multivariate analysis of GOG-99 H-IR patients, factors significant for PFS were $250 \%$ depth of invasion (hazard ratio $[\mathrm{HR}]=8.01,95 \%$ CI: $1.48-44.16 ; \mathrm{p}=0.017$ ), grade 3 disease (HR 1.99, 95\% CI: 1.14-3.49; $\mathrm{p}=0.016$ ), nonwhite race (HR 1.62, 95\% CI: 1.02-2.89; $\mathrm{p}=0.043$ ), age (HR 1.05, 95\% CI: 1.03-1.07; p<0.001), and BMI (HR 1.03, 95\% CI: $1.01-$ $1.05 ; \mathrm{p}=0.008$ ). For OS these same factors remained significant except depth of invasion lost significance and presence of LVSI (HR 1.93, 95\% CI: 1.19-3.15; $\mathrm{p}=0.008$ ) was added. Meeting GOG-99 H-IR criteria, stage, and receiving adjuvant therapy did not independently predict PFS or OS. The exclusion of advanced stage and high risk histologies likely accounts for the overall low rate of disease recurrence in this subset, with 5.4\% recurrence in 70-79yo compared to $14.6 \%$ in the same age group for the total LAP2 population. The 70 mo. PFS for $<70$ yo vs. $\geq 70 y o$ is $88 \%$ vs. $78 \%$ (p < 0.001 ). The $70 \mathrm{mo}$. OS for $<70$ yo vs. $\geq 70 y o$ is $93 \%$ vs. $83 \%(\mathrm{p}<0.001)$ (Figure 2$)$.

\section{Recurrence by number of H-IR risk factors in GOG99 H-IR population > 70yo}

In order to further explore the contribution of age on risk for recurrence, patients $>70$ yo were divided into those who met GOG-99 H-IR criteria due to age >70yo and one other uterine factor and those who met GOG-99 H-IR criteria by age $>70$ yo and 2 or 3 uterine factors (i.e.: this group met H-IR criteria even if they were 50-70yo). Of the 361 patients > 70yo who met GOG-99 H-IR criteria the majority (59\%) met criteria by age and grade 2 or 3 disease. The recurrence rate in this group was 3.3\% (7/213). All other groups that met GOG-99 H-IR criteria by age $>70$ yo and one uterine factor had a $0 \%$ recurrence rate, although numbers are low. Patients who met H-IR criteria by age > 70yo and two uterine factors had a risk of recurrence of $0 \%$ to $7.6 \%$ with the highest in patients with grade 2 or 3 disease, $250 \%$ depth of invasion, and negative LVSI. Finally, patients $>70$ yo with all three uterine risk factors $(n=43)$ had the highest risk of disease recurrence at $20.9 \%$ (Table 5).

\section{DISCUSSION}

Results from this analysis show that in a healthy, clinically early stage, fully staged endometrial cancer population, patients $\geq 70$ yo have worse PFS and OS than patients <70yo despite same surgical management and similar rates of adjuvant therapy.

Retrospective studies have investigated the reason for worse outcomes in older endometrial cancer patients. A Surveillance, Epidemiology, and End Results Program retrospective analysis of over 37,000 women with endometrial cancer evaluated differences in surgical management and adjuvant therapy and found that $95 \%$ of women $<70$ yo underwent a hysterectomy while only $2 / 3$ of patients $>80$ yo did. For women $65-69$ yo, $43 \%$ underwent lymph node dissection, and only $25 \%$ of women $>85$ yo. Patients $>70$ yo had higher grade and stage disease. This analysis found that older women were less likely to receive adjuvant radiation therapy. Cancer specific mortality increased with age with women $\$ 80$ yo more likely to die of endometrial cancer than women 65-69yo. This analysis suggests that worse disease-free survival seen in elderly patients is attributable to less aggressive primary and 
adjuvant therapies compared to younger patients [16]. Importantly, this study involves a different patient population than the current study.

Our results suggest that age and high risk uterine factors account for worse survival in older endometrial cancer patients. Patients $>70$ yo had higher rates of serous histology, high grade disease, greater depth of invasion, and LVSI. These uterine factors, along with age, are associated with worse survival in multivariate analysis. Previous studies have found that serous carcinoma is associated with poor prognosis and occurs more frequently in older patients. Although only $10 \%$ of all endometrial cancers are serous histology, it accounts for $39 \%$ of endometrial cancer deaths [17]. Studies show that up to $22 \%$ of endometrial cancers diagnosed in women $>75$ yo are serous cancers, compared to only $3 \%$ in women less than 45 yo [18]. In this study $20 \%$ of patients $70-79$ yo had serous histology, compared to only $3 \%$ of patients $<50$ yo.

Although older patients in this study had higher rates of lymph node metastasis, this was not an independent predictor for PFS or OS. We have shown this in a previous study where 352 clinical stage 1 endometrial cancer patients were divided into GOG-99 and PORTEC1 H-IR criteria and uterine factors for recurrence, lymph node metastasis, and recurrence rates were compared. When uterine factors and lymph node status were both examined for ability to predict recurrence, uterine factors were more important than nodal status [19]. In a retrospective chart review of patients with pelvic lymphadenectomy (an average of 6 lymph nodes removed) and para-aortic lymphadenectomy (12\% of patients), factors independently associated with increased risk of death included high risk uterine factors but not pelvic node status when uterine factors were taken into account [20]. In the LAP2 study the median pelvic and paraaortic lymph node count was 18 and 7 in the laparotomy group and 17 and 7 in the laparoscopy group.

When looking at the GOG-99 H-IR group; a truly early stage, node negative population with endometrioid histology; older patients received similar treatment and had similar, and low, recurrence rates compared to younger patients who met the same H-IR criteria. The only exception being patients $<50$ yo $(n=15)$ who had higher rates of adjuvant therapy compared to other ages. Older patients had worse survival, but cause of death was mainly causes unrelated to disease. Receiving adjuvant treatment did not significantly change PFS or OS and, in fact, H-IR patients who received adjuvant treatment had higher rates of disease recurrence than H-IR patients who did not receive adjuvant therapy. The number of patients who recurred in these groups was overall low; 28 recurrences in the H-IR group without adjuvant therapy versus 24 recurrences in the H-IR group with adjuvant therapy; limiting conclusions that can be drawn from this data. Site of recurrence was similar between these groups as well. On multivariate analysis of the GOG-99 H-IR population, age and uterine factors such as tumor grade and +LVSI were predictors for survival. Details about medical comorbidities and toxicity from therapy, which are often more common in older patients, were not included in this analysis. This is a weak point of this study and could affect PFS and mortality, from other causes and from disease.

As expected with the exclusion of serous histology and occult advanced disease recurrence rates were lower in 70-79yo who met GOG-99 H-IR criteria than in the same age group in 
the total LAP2 population (5.4\% vs 14.6\%). The GOG-99 H-IR group from this study had lower than expected recurrence rates compared to the GOG-99 results where the H-IR patients who received no adjuvant therapy had recurrence rates of $26 \%$ compared to $4.4 \%$ in the same population in this study, bringing to question the true impact age has on risk of recurrence [3].

Although multivariate analysis shows that age is an independent predictor for survival, our results also show that patients $>70$ yo most commonly meet GOG-99 H-IR criteria by only one uterine risk factor (grade 2 or 3 disease, $250 \%$ depth of invasion, or presence of LVSI) and are actually at very low risk (0-3.3\%) for recurrence compared to those >70yo with multiple risk factors where risk of recurrence is as high as $21 \%$, which is helpful for guiding clinical decision-making.

Conclusions that can be drawn from looking at the risk models in this population are that older patients who are truly early stage, with endometrioid histology (GOG-99) are low risk even if they meet H-IR criteria for recurrence and may be too low risk to benefit from adjuvant therapy. In this study patients with occult stage 3 and 4 disease, as well as serous histology, likely account for the majority of recurrences, making this the group who actually might benefit from adjuvant therapy, although standard of care is controversial.

It is also important to note that in all subsets analyzed, as age increased, patients more frequently died of causes unrelated to disease or treatment. Life expectancy for women at birth in the US is 80.8 years, but once a woman reaches age 70, life expectancy increases with an expected 16 more years of life [21]. Frequently endometrial cancer patients have multiple medical comorbidities which result in shorter life expectancy. Learning how to better assess comorbidities and their effect on life expectancy could allow physicians to tailor therapy more effectively.

\section{Supplementary Material}

Refer to Web version on PubMed Central for supplementary material.

\section{Acknowledgments}

This study was supported by National Cancer Institute grants to the Gynecologic Oncology Group Administrative Office (CA 27469), the Gynecologic Oncology Group Statistical Office (CA 37517), NRG Oncology Group Grant 1 U10 CA180822 and NRG Oncology Operations Grant U10CA180868. The following Gynecologic Oncology Group member institutions participated in the primary treatment studies: Abington Memorial Hospital, Walter Reed Army Medical Center, University of Minnesota Medical School, University of Mississippi Medical Center, University of Pennsylvania Cancer Center, University of California at San Diego, University of Iowa Hospitals and Clinics, University of Texas Southwestern Medical Center at Dallas, Indiana University School of Medicine, University of California Medical Center at Irvine, Tufts-New England Medical Center, Rush-Presbyterian-St. Luke's Medical Center, University of New Mexico, The Cleveland Clinic Foundation, State University of New York at Stony Brook, Washington University School of Medicine, Memorial Sloan-Kettering Cancer Center, Columbus Cancer Council, MD Anderson Cancer Center, University of Massachusetts Medical School, Women's Cancer Center, University of Oklahoma, Tacoma General Hospital, Tampa Bay Cancer Consortium, Gynecologic Oncology Network, Fletcher Allen Health Care, University of Wisconsin Hospital, Women and Infants Hospital, and CCOP.

\section{REFERENCES}

1. Siegel RL, Miller KD, Jemal A. Cancer statistics, 2016. CA: a Cancer Journal for Clinicians. 2016; 66(1):7-30. [PubMed: 26742998] 
2. American Cancer Society. Cancer Facts \& Figures 2012. American Cancer Society; Atlanta: 2012.

3. Keys HM, et al. A phase III trial of surgery with or without adjunctive external pelvic radiation therapy in intermediate risk endometrial adenocarcinoma: a Gynecologic Oncology Group study. Gynecologic Oncology. 2004; 92(3):744-51. [Erratum appears in Gynecol Oncol. 2004 Jul;94(1): 241-2]. [PubMed: 14984936]

4. Creutzberg CL, et al. Surgery and postoperative radiotherapy versus surgery alone for patients with stage-1 endometrial carcinoma: multicentre randomised trial. PORTEC Study Group. Post Operative Radiation Therapy in Endometrial Carcinoma. Lancet. 2000; 355(9213):1404-11. [PubMed: 10791524]

5. Walker JL, et al. Laparoscopy compared with laparotomy for comprehensive surgical staging of uterine cancer: Gynecologic Oncology Group Study LAP2. Journal of Clinical Oncology. 2009; 27(32):5331-6. [PubMed: 19805679]

6. Walker JL, et al. Recurrence and survival after random assignment to laparoscopy versus laparotomy for comprehensive surgical staging of uterine cancer: Gynecologic Oncology Group LAP2 Study. Journal of Clinical Oncology. 2012; 30(7):695-700. [Erratum appears in J Clin Oncol. 2012 May 1;30(13):1570]. [PubMed: 22291074]

7. Pearson K. On the criterion that a given system of deviations from the probable in the case of a correlated system of variables is such that it can be reasonably supposed to have arisen from random sampling. Philosophical Magazine1900. :157-175.

8. Whitney D, Mann H. On a test of whether one of two random variables is stochastically larger than the other. Ann Math Stat. 1947; 18:50-60.

9. Kruskal W, Wallis W. Use of ranks in one-criterion variance analysis. J Am Stat Assoc. 1952; 47:583-621.

10. Kaplan E, Meier P. Nonparametric estimation from incomplete observations. J Am Stat Assoc. 1958; 53:457-481.

11. Cox D. Regression models and life-tables. J R Stat Soc Series B Stat Methodol. 1972; 34:187-220.

12. Harrell, F. Regression Modeling Strategies, with Applications to Linear Models, Survival Analysis and Logistic Regression. Springer; New York: 2001.

13. Marquardt D. Generalized Inverses, Ridge Regression, Biased Linear Estimation, and Nonlinear Estimation. Technometrics. 1970; 12:591-612.

14. R Core Team. A Language and Environment for Statistical Computing, R Foundation for Statistical Computing. 2013. [cited 2013; Available from: http://www.R-project.org/

15. Pecorelli S. Revised FIGO staging for carcinoma of the vulva, cervix, and endometrium. International Journal of Gynaecology \& Obstetrics. 2009; 105(2):103-4. [Erratum appears in Int J Gynaecol Obstet. 2010 Feb;108(2):176]. [PubMed: 19367689]

16. Wright JD, et al. Endometrial cancer in the oldest old: Tumor characteristics, patterns of care, and outcome. Gynecologic Oncology. 2011; 122(1):69-74. [PubMed: 21429570]

17. Ueda SM, et al. Trends in demographic and clinical characteristics in women diagnosed with corpus cancer and their potential impact on the increasing number of deaths. American Journal of Obstetrics \& Gynecology. 2008; 198(2):218.e1-6. [PubMed: 18226630]

18. Lachance JA, et al. The effect of age on clinical/pathologic features, surgical morbidity, and outcome in patients with endometrial cancer. Gynecologic Oncology. 2006; 101(3):470-5. [PubMed: 16413048]

19. Nugent EK, et al. Do uterine risk factors or lymph node metastasis more significantly affect recurrence in patients with endometrioid adenocarcinoma? Gynecologic Oncology. 2012; 125(1): 94-8. [PubMed: 22155415]

20. Kwon JS, et al. Are uterine risk factors more important than nodal status in predicting survival in endometrial cancer? Obstetrics \& Gynecology. 2009; 114(4):736-43. [PubMed: 19888029]

21. 2009. http://www.ssa.gov/oact/STATS/table4c6.html 

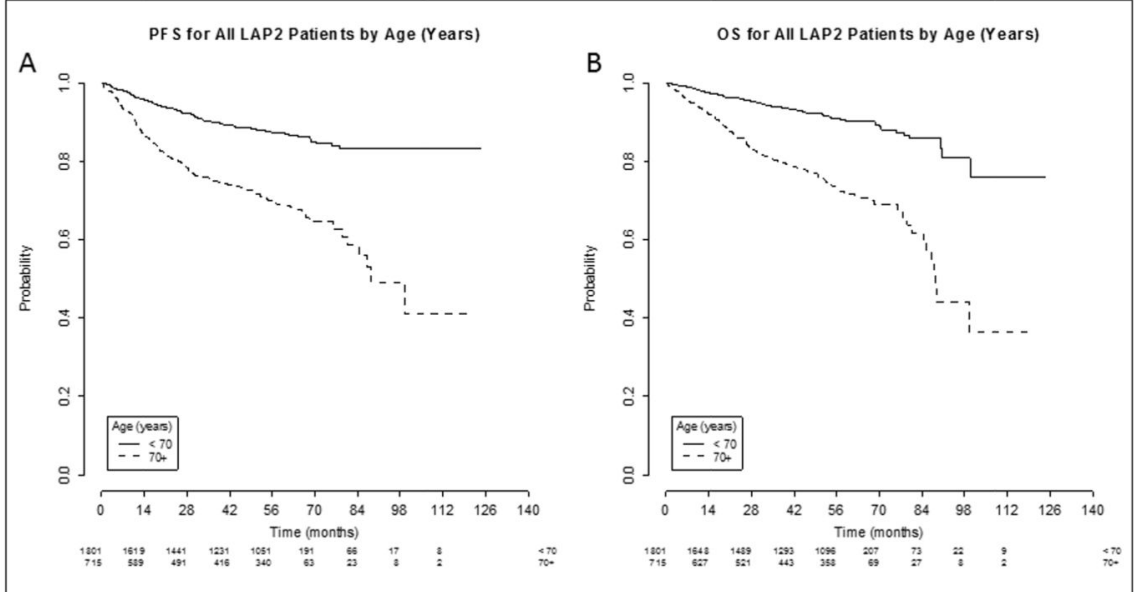

Figure 1.

Kaplan-Meier estimates of (A) progression-free survival and (B) overall survival for patients $<70$ years-old and $\geq 70$ years-old in the total LAP2 population. The 70 -month progressionfree survival for $<70$ years-old vs. $\geq 70$ years-old is $85 \%$ vs. $65 \%$ ( $p<0.001)$. The 70 -month overall survival for patients $<70$ years-old vs. $\geq 70$ years-old is $89 \%$ vs. $69 \%(\mathrm{p}<0.001)$. Pvalues are for the log-rank tests comparing the pairs of survival curves. 


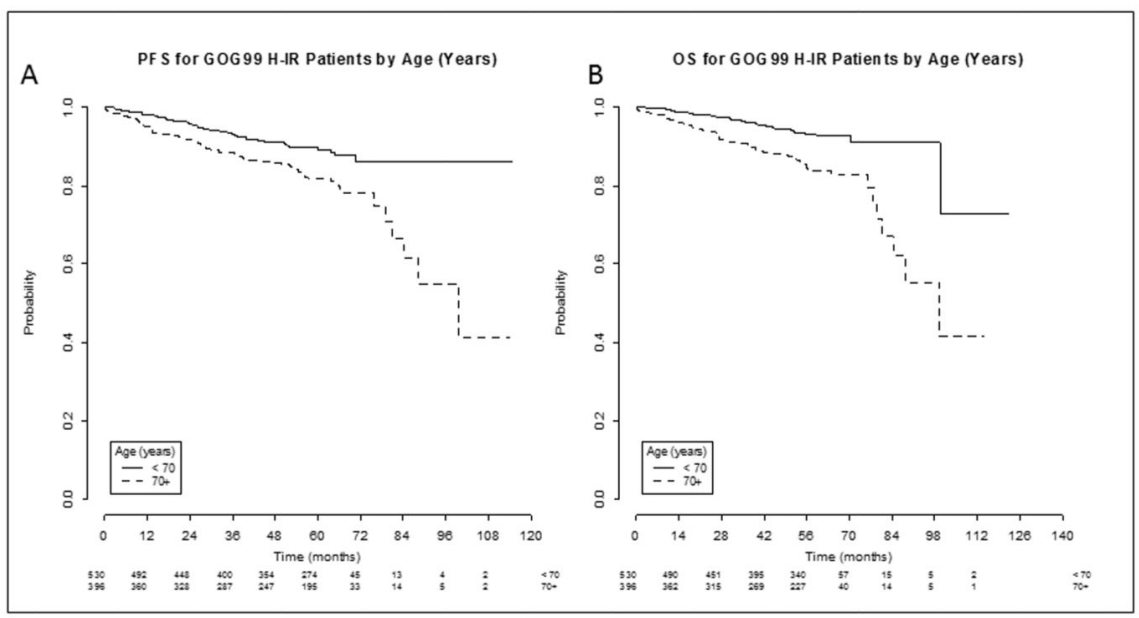

Figure 2.

Kaplan-Meier estimates of (A) progression-free survival and (B) overall survival for patients $<70$ years-old and $\geq 70$ years-old in the GOG99 High-Intermediate Risk population. The 70month progression-free survival for $<70$ years-old vs. $\geq 70$ years-old is $88 \%$ vs. $78 \%$ (p < 0.001 ). The 70-month overall survival for $<70$ years-old vs. $\geq 70$ years-old is $93 \%$ vs. $83 \%$ (p $<0.001)$. P-values are for the log-rank tests comparing the pairs of survival curves. 
Table 1

LAP2 pathology findings and outcomes by age (years) $n=2516$

\begin{tabular}{|c|c|c|c|c|c|c|}
\hline & $\begin{array}{c}<50 \\
\mathbf{n}=285 \\
\%(\mathbf{n})\end{array}$ & $\begin{array}{c}\mathbf{5 0 - 5 9} \\
\mathbf{n}=\mathbf{7 5 4} \\
\%(\mathbf{n})\end{array}$ & $\begin{array}{c}60-69 \\
n=762 \\
\%(n)\end{array}$ & $\begin{array}{c}70-79 \\
\mathrm{n}=555 \\
\%(\mathrm{n})\end{array}$ & $\begin{aligned} & \geq 80 \\
\mathrm{n} & =160 \\
\% & (\mathrm{n})\end{aligned}$ & $\begin{array}{c}\text { Test } \\
\text { statistic }\end{array}$ \\
\hline Histology & & & & & & $\mathrm{P}<0.001$ \\
\hline Endometrioid & $92.3(263)$ & $87.9(663)$ & $79(602)$ & $71.2(395)$ & $60(96)$ & \\
\hline serous & $2.8(8)$ & $5.7(43)$ & $11.4(87)$ & $19.5(108)$ & $26.9(43)$ & \\
\hline other & $4.9(14)$ & $6.4(48)$ & $9.6(73)$ & $9.4(52)$ & $13.1(21)$ & \\
\hline \multicolumn{6}{|l|}{ FIGO surgical stage } & $\mathrm{P}<0.001$ \\
\hline IA & $78.9(224)$ & $75.0(561)$ & $70.1(531)$ & $59.7(331)$ & $53.5(85)$ & \\
\hline IB & $6.0(17)$ & $8.8(66)$ & $12.4(94)$ & $18.6(103)$ & $21.4(34)$ & \\
\hline II & $6.3(18)$ & $3.3(25)$ & $3.6(27)$ & $3.4(19)$ & $6.3(10)$ & \\
\hline IIIC1 & $3.2(9)$ & $4.7(35)$ & $4.1(31)$ & $6.3(35)$ & $4.4(7)$ & \\
\hline IIIC2 & $2.8(8)$ & $4.1(31)$ & $4.1(31)$ & $5.1(28)$ & $6.9(11)$ & \\
\hline IVB & $0.7(2)$ & $1.7(13)$ & $3.2(24)$ & $3.1(17)$ & $6.9(11)$ & \\
\hline \multicolumn{6}{|l|}{ Tumor grade } & $\mathrm{P}<0.001$ \\
\hline 1 & 33.7 (96) & $26.3(198)$ & $19.4(148)$ & $13.0(72)$ & $13.1(21)$ & \\
\hline 2 & $47.7(136)$ & $49.5(373)$ & $47.2(360)$ & $40.2(223)$ & $31.9(51)$ & \\
\hline 3 & $17.2(49)$ & $22.5(170)$ & $29.3(223)$ & 42.7 (237) & $50.6(81)$ & \\
\hline Not graded & $1.4(4)$ & $1.7(13)$ & $4.1(31)$ & $4.1(23)$ & $4.4(7)$ & \\
\hline \multicolumn{6}{|l|}{ Myometrial invasion } & $\mathrm{P}<0.001$ \\
\hline none & $14.2(40)$ & $9.6(72)$ & $7.9(60)$ & $4.7(26)$ & $3.8(6)$ & \\
\hline endometrial & $41.8(118)$ & $34.1(256)$ & $30.2(228)$ & $24.6(136)$ & $18.2(29)$ & \\
\hline$<\mathbf{5 0 \%}$ & $29.8(84)$ & $38.7(291)$ & $39.3(297)$ & $36.6(202)$ & $37.7(60)$ & \\
\hline$\geq \mathbf{5 0 \%}$ & $12.4(35)$ & $16.1(121)$ & $20.4(154)$ & $29.7(164)$ & $36.5(58)$ & \\
\hline+ LVSI & $13.5(38)$ & $17.4(129)$ & $19.5(146)$ & $24.5(134)$ & $27.2(43)$ & $\mathrm{P}<0.001$ \\
\hline$+\mathbf{L N}$ & $7.0(20)$ & $9.4(71)$ & $9.3(71)$ & $12.3(68)$ & $13.1(21)$ & $\mathrm{P}=0.079$ \\
\hline \multicolumn{7}{|l|}{ Postoperative therapy } \\
\hline chemotherapy & $8.8(25)$ & $9.8(74)$ & $12.3(94)$ & $13.5(75)$ & $10.0(16)$ & $\mathrm{P}=0.122$ \\
\hline radiation & $20.0(57)$ & $21.5(162)$ & $24.0(183)$ & $30.6(170)$ & $23.8(38)$ & $P=0.001$ \\
\hline none & 73.3 (209) & $71.4(538)$ & $66.4(506)$ & $60.2(334)$ & $64.4(103)$ & $\mathrm{P}<0.001$ \\
\hline Any & $22.8(65)$ & $25.9(195)$ & $29.1(222)$ & $36.9(205)$ & $29.4(47)$ & $\mathrm{P}<0.001$ \\
\hline Recurrence & $4.9(14)$ & $6.6(50)$ & $10.5(80)$ & $14.6(81)$ & $14.4(23)$ & $\mathrm{P}<0.001$ \\
\hline 5-year OS & 96.8 & 91.9 & 86.5 & 76.7 & 53.2 & $\mathrm{P}<0.001^{\dagger}$ \\
\hline Cause of death $(n=345)$ & & & & & & $\mathrm{P}<0.007$ \\
\hline disease & $62.5(5)$ & $71.7(38)$ & $63.9(62)$ & $52.4(65)$ & $41.3(26)$ & \\
\hline other & $37.5(3)$ & $28.3(15)$ & $36.1(35)$ & $47.6(59)$ & $58.7(37)$ & \\
\hline
\end{tabular}

Int J Gynecol Cancer. Author manuscript; available in PMC 2018 May 01. 
Abbreviations: FIGO, International Federation of Gynecology and Obstetrics; LVSI, Lymphovascular Space Invasion; LN, lymph node; OS, Overall survival.

Single-line binary variables do not list their opposing category.

Variables were compared between the patient subgroups by the Pearson chi-square test.

${ }^{\dagger}$ The p-value is from the log-rank test of survival differences among the age subgroups. 
Table 3

Patient Characteristics by Age (GOG 99 H-IR group, $\mathrm{n}=926$ )

\begin{tabular}{|c|c|c|c|c|c|c|}
\hline & $\begin{array}{c}<50 \\
N=15 \\
\%(n)\end{array}$ & $\begin{array}{c}\mathbf{5 0}-\mathbf{5 9} \\
\mathbf{N}=\mathbf{2 5 4} \\
\%(\mathbf{n})\end{array}$ & $\begin{array}{c}60-69 \\
\mathbf{N}=261 \\
\%(n)\end{array}$ & $\begin{array}{c}70-79 \\
\mathbf{N}=315 \\
\%(n)\end{array}$ & $\begin{array}{c}\geq 80 \\
\mathrm{~N}=81 \\
\%(\mathrm{n})\end{array}$ & $\begin{array}{c}\text { Test } \\
\text { Statistic }\end{array}$ \\
\hline Postoperative therapy & & & & & & $\mathrm{P}<0.001$ \\
\hline chemotherapy & $20.0(3)$ & $2.0(5)$ & $2.3(6)$ & $2.5(8)$ & $1.2(1)$ & $\mathrm{P}<0.001$ \\
\hline radiation & $60.0(9)$ & $27.2(69)$ & $27.2(71)$ & $24.4(77)$ & $17.3(14)$ & $P=0.011$ \\
\hline none & $33.3(5)$ & $68.5(174)$ & $70.1(183)$ & $70.5(222)$ & $71.6(58)$ & $P=0.046$ \\
\hline Recurrence & $20.0(3)$ & $4.3(11)$ & $6.1(16)$ & $5.4(17)$ & $7.4(6)$ & $P=0.128$ \\
\hline 5-yr OS & 91.6 & 94.7 & 90.6 & 88.1 & 65 & $\mathrm{P}<0.001^{\dagger}$ \\
\hline Cause of death $(n=923)$ & & & & & & $\mathrm{P}<0.001$ \\
\hline alive & $93.3(14)$ & $95.7(243)$ & $91.6(239)$ & $88.5(278)$ & $70.9(56)$ & \\
\hline disease & $6.7(1)$ & $3.1(8)$ & $4.2(11)$ & $3.2(10)$ & $7.6(6)$ & \\
\hline Other & $0.0(0)$ & $1.2(3)$ & $4.2(11)$ & $8.3(26)$ & $21.5(17)$ & \\
\hline
\end{tabular}

Abbreviations: GOG99, Gynecologic oncology group protocol 99; H-IR, high-intermediate risk.

Variables were compared between the patient subgroups by the Pearson chi-square test.

The p-value is from the log-rank test of survival differences among the age subgroups. 


\section{Table 4}

Recurrence and survival in GOG-99 H-IR patients with and without adjuvant therapy

\begin{tabular}{lccc}
\hline & $\begin{array}{c}\text { Untreated } \\
\mathbf{N = 6 4 2} \\
\mathbf{\%}(\mathbf{n})\end{array}$ & $\begin{array}{c}\text { Treated } \\
\mathbf{N = 2 5 0} \\
\mathbf{\%}(\mathbf{n})\end{array}$ & $\begin{array}{c}\text { Test } \\
\text { Statistic }^{*}\end{array}$ \\
\hline Recurrence & $4.4(28)$ & $9.6(24)$ & $\mathrm{P}<0.003$ \\
2-yr PFS & 95.9 & 91.4 & $\mathrm{P}=0.16^{\dagger}$ \\
5-yr OS & 90.3 & 87.9 & $\mathrm{P}=0.20^{\dagger}$ \\
\hline
\end{tabular}

Abbreviations: GOG-99, Gynecologic Oncology Group protocol 99; H-IR, high-intermediate risk; PFS, progression-free survival; OS, overall survival.

Variables were compared between the patient subgroups by the Pearson chi-square test.

${ }^{\dagger}$ The p-value is from the log-rank test of survival differences between the treatment subgroups. 


\section{Table 5}

GOG99 H-IR patients $>70$ years old by uterine factors

\begin{tabular}{|c|c|c|}
\hline GOG 99 H-IR (>70yo) & $\mathbf{N}=361$ & $\begin{array}{l}\text { Recurrence rate } \\
\% \text { (n) }\end{array}$ \\
\hline \multicolumn{3}{|l|}{ H-IR by 1 uterine factor } \\
\hline Grade 2 or 3 & 213 & $3.3(7)$ \\
\hline +LVSI & 5 & $0(0)$ \\
\hline$\triangle 0 \%$ depth of invasion & 11 & $0(0)$ \\
\hline \multicolumn{3}{|l|}{ H-IR by 2 uterine factors } \\
\hline +LVSI; $\leq 50 \%$ depth of invasion & 4 & $0(0)$ \\
\hline Grade 2 or 3 ; +LVSI & 19 & $5.3(1)$ \\
\hline Grade 2 or $3 ; \leq 50 \%$ depth of invasion & 66 & $7.6(5)$ \\
\hline
\end{tabular}

H-IR by 3 uterine factors

Grade 2 or 3; +LVSI; $\mathbf{5 0 \%}$ depth of invasion $\quad 43 \quad 20.9(9)$

Abbreviations: GOG99, Gynecologic oncology group protocol 99; H-IR, high-intermediate risk; LVSI, Lymphovascular Space Invasion. 